\title{
INFLUÊNCIA DA TEMPERATURA DE SECAGEM EM LEITO DE JORRO DE PASTA DE VEGETAIS ENRIQUECIDA DE PLASMA BOVINO NAS CARACTERÍSTICAS DO PRODUTO EM PÓ
}

\author{
H. H. L. ZACCARDI ${ }^{1}$, J. V. O. CASTRO ${ }^{1}$, T. MÜLLER ${ }^{1}$, S. F. ROCHA ${ }^{1}$ e L. A. A. PINTO ${ }^{1}$ \\ ${ }^{1}$ Universidade Federal do Rio Grande, Escola de Química e Alimentos \\ E-mail para contato: heraldozacardi@gmail.com
}

\begin{abstract}
RESUMO - A operação de secagem em leito de jorro foi realizada nas temperaturas do ar de 90,100 e $110^{\circ} \mathrm{C}$, com a concentração de sólidos de $5 \%$ $(\mathrm{m} / \mathrm{m})$ e vazão de alimentação de pasta de $250 \mathrm{~mL} \mathrm{~h}^{-1}$. Foram realizadas a determinação de umidade, análise morfológica e cinética de reidratação. Para todos os experimentos foram encontrados valores de umidade do produto final abaixo do valor de $10 \%$ (base úmida). As imagens de MEV da pasta vegetal e dos pós obtidos apresentaram estruturas compactas e rígidas, que podem ser atribuídas à presença de fibras. Os produtos em pó apresentaram-se homogêneos com partículas de superfície irregular, tendo tamanhos de partícula menores que 100 $\mu \mathrm{m}$. Para a constante de reidratação dos produtos em pós foram encontrados maiores valores nas temperaturas de secagem mais elevadas.
\end{abstract}

\section{INTRODUÇÃO}

A secagem de alimentos é comumente utilizada a fim de reduzir o teor de umidade, e consequentemente, aumentar o tempo de conservação do produto seco, através do impedimento de reações microbiológicas (Mujumdar e Huang, 2007). A crescente demanda por alimentos desidratados requer a otimização das condições da operação de secagem, com a finalidade de melhorar a eficiência do processo e a qualidade final do produto seco (Banga et al., 2003).

Além disso, a técnica de secagem em leitos móveis tem sido empregada para produção de alimentos na forma de pós. No entanto, o produto obtido geralmente apresenta-se como um pó fino com uma baixa dispersão e solubilização em fase líquida, tendo ainda outros problemas causados pela granulometria como a compactação e a difícil escoabilidade das partículas durante o armazenamento e manipulação (Martins e Kieckbusch, 2008). Outros estudos afirmam também que altos teores de umidade do pó podem ser relacionados à perda de qualidade e a degradação do produto, e ainda a instantaneidade do pó é atribuída à morfologia da partícula (Braga e Rocha, 2013).

Perante a dificuldade de dispersão e solubilização dos pós finos, é necessário que sejam efetuados estudos sobre a capacidade de reidratação dos pós. Tal técnica tem como principal característica apresentar um comportamento oposto ao da operação secagem. A reidratação de tecidos vegetais secos é composta por três etapas: a inibição de água do material, alteração de 
volume das partículas (inchamento) e lixiviação de alguns compostos solúveis, sendo que o inchamento é frequentemente proporcional à quantidade de água absorvida (Krokida e Marinos-Kouris).

O trabalho teve como objetivo avaliar a influência da temperatura de secagem nas propriedades dos produtos em pó, como umidade, morfologia e tempo de reidratação, obtidos pasta de vegetais enriquecida com plasma bovino seca em leito de jorro cônico.

\section{MATERIAL E MÉTODOS}

\subsection{Matéria-prima, Formulação e Obtenção da Pasta de Vegetais Enriquecida com Plasma Bovino}

Para obtenção da pasta os vegetais utilizados foram: cebola (Allium cepa L.), tomate (Lycopersicum esculentum), cenoura (Daucus carota L.), couve (Brassica oleracea), batata (Solanum tuberosum), óleo de soja comercial e plasma bovino. O sangue bovino necessário para obtenção do plasma foi coletado em um abatedouro situado na cidade de Pelotas/RS com as práticas de assepsia devidamente empregadas. A extração do plasma bovino foi de acordo com Lopes et al., (2008). Os vegetais e o óleo de soja foram adquiridos em feiras livres e nos mercados da cidade do Rio Grande/RS, respectivamente.

A formulação da pasta de vegetais foi determinada utilizando a técnica de programação linear de acordo com trabalho anterior (Rocha et al., 2014), otimizando a maximização do conteúdo proteico através de uma função objetivo, sendo utilizado um conjunto de restrições para o conteúdo de carboidratos, proteínas e lipídios, e valor calórico. A Figura 1 mostra o fluxograma do processamento da pasta de vegetais

Figura 1 - Fluxograma do processamento da pasta de vegetais enriquecida de plasma bovino.

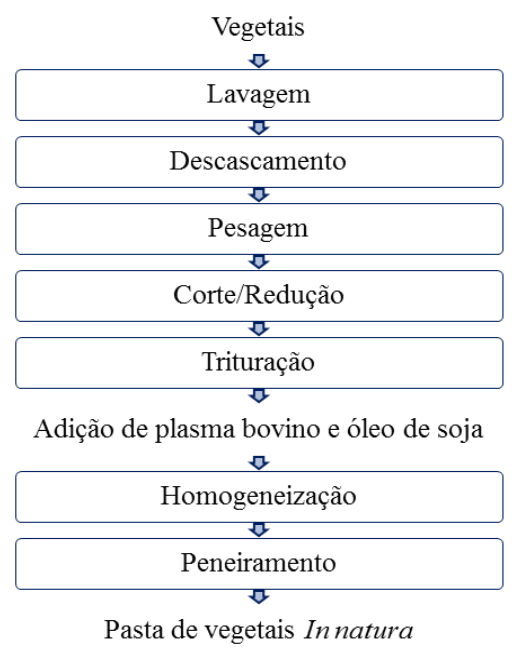

\subsection{Secagem em Leito de Jorro}

A secagem foi realizada em célula de geometria cônica, com o diâmetro de $17,5 \mathrm{~cm}$, com extremidades inferior e superior de vidro com ângulo de $60^{\circ}$ e altura de $15 \mathrm{~cm}$ cada, com 
diâmetro de entrada de 2,9 cm e a relação (Dc/ Di) igual a 6,0. O leito de inertes foi composto por $0,5 \mathrm{~kg}$ de partículas de polietileno com diâmetro médio de $3,2 \mathrm{~mm}$, esfericidade $0,7 \mathrm{e}$ densidade de $0,96 \mathrm{~g} \mathrm{~cm}^{-3}$. O fluido utilizado na secagem foi ar aquecido por um conjunto de três resistências de $800 \mathrm{~W}$ cada. A vazão de ar foi determinada por placa de orifício. Foram fixadas a pressão de atomização em $200 \mathrm{kPa}$ absoluta e a taxa de circulação de sólidos de $100 \%$ acima da velocidade de jorro mínimo, como é indicado na literatura para secagem de pastas e suspensões (Epstein e Grace, 2011).

A pasta teve a sua concentração inicial ajustada com água para atingir $5 \%(\mathrm{~m} / \mathrm{m})$ de sólidos, e foi alimentada no leito de forma semicontínua, por meio de um jato pistonado a uma vazão de $250 \mathrm{~mL} \mathrm{~h}^{-1}$, nas temperaturas de 90,100 e $110^{\circ} \mathrm{C}$. O regime da operação foi monitorado pelas medidas das temperaturas do ar de entrada e saída da célula de secagem das temperaturas de bulbo seco e de bulbo úmido do ar a cada 2 min até atingir o leito entrar em regime (30 min), e após a cada 5 min durante a operação. O tempo total de operação para cada experimento de secagem foi de $6 \mathrm{~h}$, e o pó seco foi recolhido em um recipiente de vidro acoplado a um ciclone.

\subsection{Métodos Analíticos}

Determinação de Umidade: A umidade foi determinada segundo a metodologia $\mathrm{n}^{\circ}$ 013/IV, utilizando uma estufa a vácuo à $100 \mathrm{mmHg}$ e $70^{\circ} \mathrm{C}$ por $6 \mathrm{~h}$, segundo IAL (2004).

Microscopia Eletrônica de Varredura: As amostras foram analisadas para a determinação da estrutura morfológica por de microscopia eletrônica de varredura (MEV) utilizando para as análises um microscópio eletrônico (Jeol, JSM 6010LV, Japão). Os pós foram metalizados com ouro em câmara de vácuo e foram utilizadas acelerações de voltagem de $15 \mathrm{kV}$ para a obtenção das imagens, com uma magnificação de 100 vezes.

Cinética de Reidratação: Os pós das amostras foram reidratados com água destilada a 25 ${ }^{\circ} \mathrm{C}$, sendo utilizadas cerca de $1,0 \mathrm{~g}$ de pó. A reidratação foi avaliada pela quantidade de água adsorvida $(0,1 \mathrm{~mL})$ pelo pó em determinado tempo para cada amostra, e os experimentos foram realizados em duplicata. As curvas foram expressas em relação do teor de umidade (base seca) e do tempo de reidratação, e a constante de reidratação (kr) foi estimada de acordo com a Equação 1.

$$
\frac{\mathrm{x}_{\mathrm{s}}-\mathrm{x}}{\mathrm{x}_{\mathrm{s}}-\mathrm{x}_{0}}=\mathrm{a} \exp ^{-\mathrm{k}_{\mathrm{r}} \mathrm{t}}
$$

onde $\left(\mathrm{X}_{\mathrm{s}}-\mathrm{X}\right)\left(\mathrm{X}_{\mathrm{s}}-\mathrm{X}_{0}\right)^{-1}$ é o adimensional de água livre, "a" é um parâmetro de ajuste, $\mathrm{kr}$ é a constante cinética de reidratação $\left(\mathrm{min}^{-1}\right)$ e t é o tempo de reidratação (min). Os parâmetros $\mathrm{X}_{\mathrm{s}}$, $\mathrm{X}$ e $\mathrm{X}_{0}$ são os teores de umidade de saturação, umidade no tempo t e umidade inicial do pó seco, respectivamente.

Análise Estatística: Os resultados foram analisados através do Teste de Tukey ao nível de $95 \%$ de confiança $(\mathrm{p}<0,05)$.

\section{RESULTADOS E DISCUSSÃO}


O conteúdo de umidade presente nos pós, obtidos pelas operações de secagem em leito de jorro cônico nas diferentes condições de operação estão apresentados na Tabela 1.

Tabela 1 - Conteúdo final de umidade do pó obtido na secagem de pasta de vegetais enriquecida de plasma bovino.

\begin{tabular}{|c|c|}
\hline Temperatura $\left({ }^{\circ} \mathrm{C}\right)$ & Umidade $($ b.u $)(\%)(\mathrm{m} / \mathrm{m})$ \\
\hline 90 & $8,9 \pm 0,5^{\mathrm{a}}$ \\
\hline 100 & $6,5 \pm 0,1^{\mathrm{b}}$ \\
\hline 110 & $4,3 \pm 0,1^{\mathrm{c}}$ \\
\hline
\end{tabular}

Conforme apresentado na Tabela 1, a técnica de secagem de pastas no leito de jorro, promove um produto com teores de umidade abaixo de $10 \%$ (b.u), além disso, a umidade foi diminuindo de acordo com o aumento da temperatura. Esse comportamento é desejável quando se tem somente como finalidade a preservação do pó. Entretanto, quando trata-se de uma posterior solubilização pós com baixos teores de umidade apresentam uma certa resistência a dispersarem na fase líquida (Martins e Kieckbusch, 2008). A Figura 2 apresenta as estruturas morfológicas das amostras em pó secas no leito de jorro cônico em diferentes temperaturas $\left(90,100\right.$ e $\left.110^{\circ} \mathrm{C}\right)$.

Figura 2 - Microscopia eletrônica de varredura $(\times 100)$ dos pós secos de pastas de vegetais enriquecida com plasma bovino nas temperaturas de (a) $90^{\circ} \mathrm{C}$, (b) $100^{\circ} \mathrm{C}$ e (c) $110^{\circ} \mathrm{C}$.
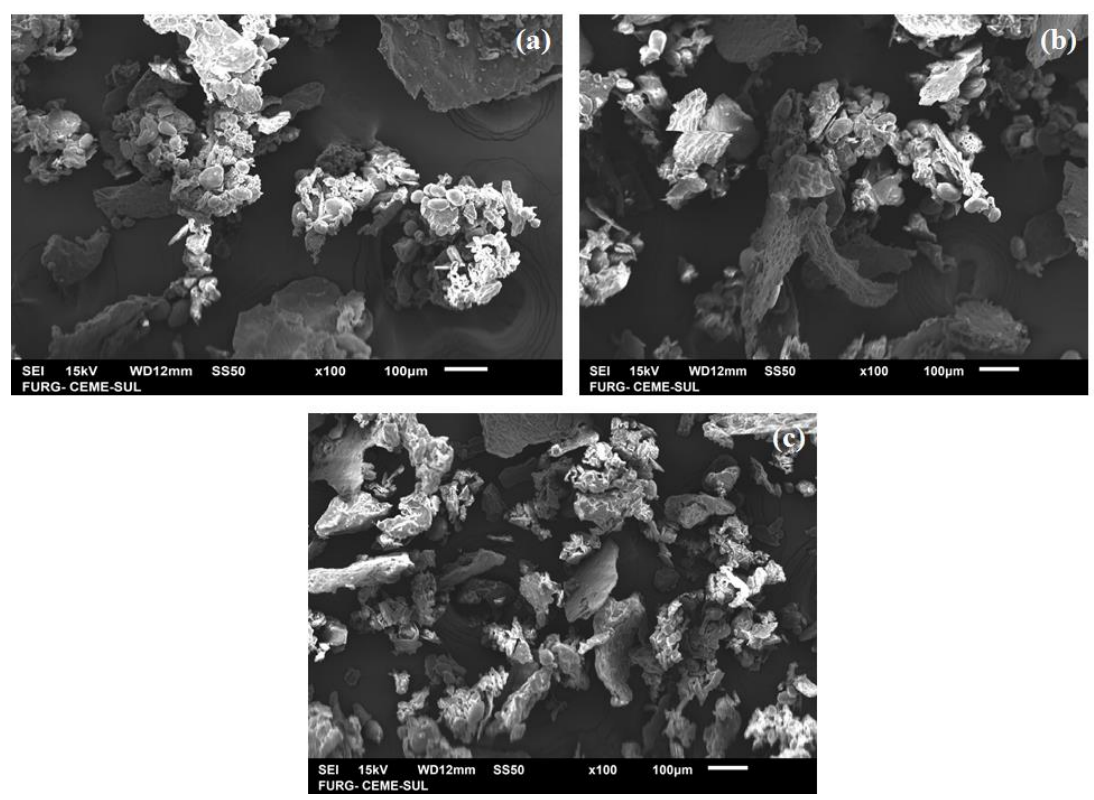

As partículas da pasta de vegetais enriquecida com plasma bovino seca em leito de jorro em diferentes temperaturas do ar apresentaram tamanhos inferiores a $100 \mu \mathrm{m}$, como é possível observar nas imagens da Figura 2. O pequeno tamanho das partículas secas pode ser associado à forma de alimentação da pasta, pois durante o processo de secagem a alimentação foi feita por atomização com ar comprimido, o que gera gotículas bem pequenas quando comparado a outras formas, como a de gotejamento por exemplo. As imagens mostram 
também que as amostras em pó apresentaram estruturas irregulares e compactas. A morfologia do floco irregular em forma partículas pode ser associada com a deformação mecânica da película depositada nas partículas de inertes devido à fricção entre as partículas e a parede do leito (Braga e Rocha, 2013).

A influência da temperatura de secagem na morfologia de partículas foi estudada por Oakley (1997) e Alamilla-Beltrán et al. (2005), os quais relatam que a secagem de pastas à baixas temperaturas produz partículas denteadas e à altas temperaturas resultam em um grande número de partículas fraturadas. Tal comportamento pode ser atribuído à umidade do produto final, pois nas maiores temperaturas obtiveram-se menores valores de umidade (conforme Tabela 1), fazendo com que a formação de aglomerados fosse minimizada como mostra a Figura 2 (b) e praticamente inexistente na Figura 2 (c). A Figura 3 apresenta as curvas de reidratação do pó seco de pasta de vegetais enriquecida de plasma bovino.

A Figura 3 apresenta as curvas de reidratação das amostras em pó secas em leito de jorro de pasta de vegetais enriquecida de plasma bovino. A figura mostra que o tempo total de reidratação variou em função da temperatura do ar utilizada na operação de secagem, pois na amostra seca em temperatura mais elevada apresentou um tempo menor para atingir o equilíbrio.

Figura 3 - Curvas de reidratação das amostras em pó de pasta de vegetais enriquecida de plasma bovino secas em leito de jorro as temperaturas do ar de 90,100 e $110{ }^{\circ} \mathrm{C}$.

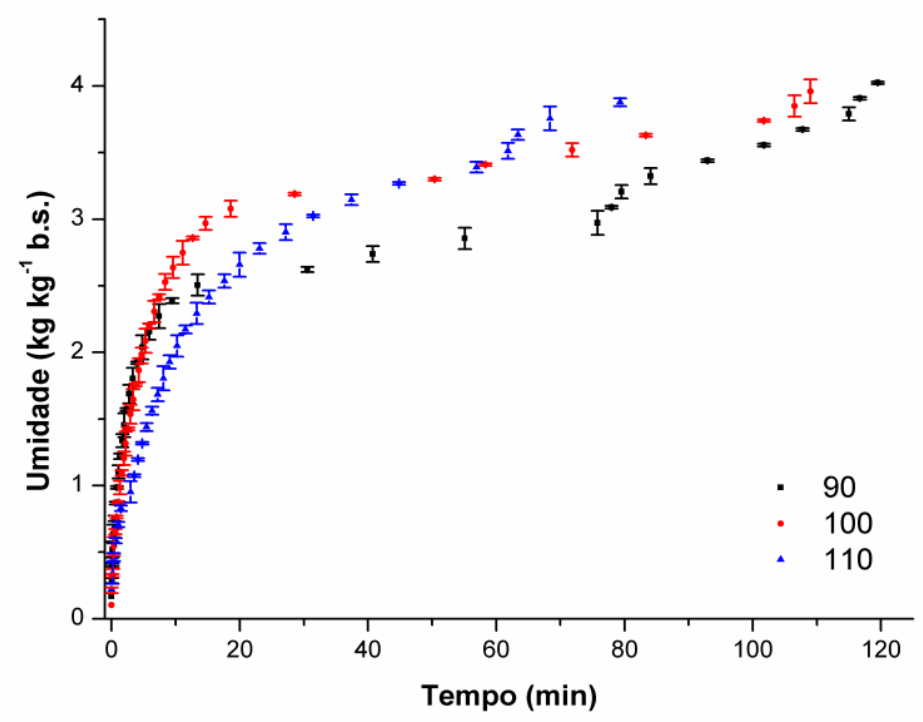

Pode-se observar também na Figura 3 que a primeira fase de reidratação está associada a uma rápida adsorção de água até atingir a umidade de transição, e esse fenômeno pode devido à capilaridade da partícula (Markowski et al. 2009). Na segunda fase ocorreu uma adsorção mais lenta de água até atingir a umidade de equilíbrio, o que correspondendo ao final da reidratação. A Tabela 2 mostra os valores da constante de reidratação (kr) obtidos para a reidratação da pasta de vegetais enriquecida de plasma bovino. Os resultados da constante de reidratação $(\mathrm{kr})$ mostraram que na temperatura de secagem mais alta $\left(110{ }^{\circ} \mathrm{C}\right)$ apresentou o valor mais alto de kr, obtendo a difusão mais rápida de água no material. 
Tabela 2 - Valores da constante de reidratação $(\mathrm{kr})$ das amostras em pó de pasta de vegetais enriquecida de plasma bovino seca em leito de jorro nas temperaturas de 90,100 e $110{ }^{\circ} \mathrm{C}$.

\begin{tabular}{|c|c|c|}
\hline Temperatura $\left({ }^{\circ} \mathrm{C}\right)$ & $\mathrm{kr}\left(\mathrm{min}^{-1}\right)$ & $\mathrm{R}^{2}$ \\
\hline 90 & $0,033 \pm 0,005^{\mathrm{a}}$ & 0,969 \\
\hline 100 & $0,045 \pm 0,002^{\mathrm{b}}$ & 0,960 \\
\hline 110 & $0,077 \pm 0,001^{\mathrm{c}}$ & 0,928 \\
\hline
\end{tabular}

\section{CONCLUSÃO}

As amostras em pó obtidas da secagem da pasta de vegetais em leito de jorro atingiram valores de umidade final menores do que $10 \%$ (b.u.), em todas as temperaturas do ar utilizadas. As microscopias dos pós secos obtidos apresentaram estruturas compactas e irregulares, com tamanhos de partículas inferiores a $100 \mu \mathrm{m}$, sendo que a formação de aglomerados diminuiu com o aumento da temperatura do ar de secagem. Os resultados da constante de reidratação dos experimentos realizados apresentaram uma difusão mais rápida na temperatura mais elevada $\left(110^{\circ} \mathrm{C}\right)$.

\section{REFERÊNCIAS}

ALAMILLA-BELTRÁN, L.; CHANONA-PÉREZ， J. J.; JIMÉNEZ-APARICIO， A. R.; GUTIÉRREZ-LÓPEZ, G. F. Description of morphological changes of particles along spray drying. J. Food Eng., v. 67, p. 179-184, 2005.

BANGA, J.; BALSA-CANTO, E.; MOLES, C.; ALONSO, A. Improving food processing using modern optimization methods. Trends Food Sc. Tech., v. 14, p. 131-144, 2003.

BRAGA, M.; ROCHA, S. C. S. Drying of milk-blackberry pulp mixture in spouted bed. Can. J. Chem. Eng., v. 91, p. 1786-1792, 2013.

KROKIDA, M. K.; MARINOS-KOURIS, D. Rehydration kinetics of dehydrated products. $J$. Food Eng., v. 57, p. 1-7, 2003.

MARKOWSKI, M.; BONDARUK, J.; BLASZCZAK, W. Rehydration behavior of vacuummicrowave-dried potato cubes. Drying Technol.. v. 27, p. 296-305, 2009.

MARTINS, P. C.; KIECKBUSCH, T. G. Influence of a lipid phase on steam jet agglomeration of maltodextrin powders. Powder Technol., v. 185, p. 258-266, 2008.

MUJUMDAR, A. S.; HUANG, L. X. Global R\&D needs in drying. Drying Technol., v. 25, p. 647-658, 2007.

OAKLEY, D. E. Produce uniform particles by spray drying. Chem. Eng. Prog., v. 10, p. 4854, 1997.

ROCHA, S. F.; RODRIGUES, M. C. K.; MONTE, M. L.; LARROSA, A. P. Q; PINTO, L. A. A. Product characteristics and quality of bovine blood-enriched dried vegetable paste. $J$. Sci. Food Agr., v. 94, p. 3255-3262, 2014. 